\title{
Formal Ontology for Participative Simulation
}

\author{
J.B.M. Goossenaerts, C. Pelletier \\ Eindhoven University of Technology, Faculty of Technology Management, The Netherlands \\ Em:J.B.M.Goossenaerts@tm.tue.nl \\ C. Reyneri \\ DataConsulteam, Turin, Italy
}

R.J. van den Berg

Baan Development, Barneveld, The Netherlands

Keywords Ontology for manufacturing, simulation, participation, enterprise modelling

\begin{abstract}
This paper reports on the first results of the Ontology Workpackage of the HUMACS/PSIM project which started on April $1^{\text {st }}$ under the IMS/EU $5^{\text {th }}$ Framework Program. After summarizing guidelines for ontology development, the PSIM choices regarding domain and related knowledge areas are presented. A draft ontology configuration schema is presented. It reflects findings from the area of enterprise reference architecture and integration methodology. The position and impact of other knowledge areas, i.e., ergonomy, socio-technical systems design, IT tools and procedures, are addressed informally.
\end{abstract}

\section{INTRODUCTION}

Today's manufacturing enterprises have to optimize their production in a highly competitive and global market place. Innovative prototypes must be turned into a manufacturable product, at a much higher speed than ever before. This trend accentuates the significance of knowledge and knowledge processing in organizations. Participation is the process which allows employees to exert some influence over their work and the conditions under which they work. Competence and capability are "both a requirement for and a consequence of participation" [13]: a requirement because participation needs a minimum level of skills in order to be effective; and a consequence because participation enhances the skills levels of those involved. Participation assumes a smooth mutual communication between 
management and employees. Information infrastructure services can support this communication by providing highly visual representations of abstract processes, that contribute to a common basis for discussions and suggestions. In this respect, simulation is the construction and use of a computer-based representation, or model, of some part of the real world as a substitute vehicle for experiment and behaviour prediction. It offers an attractive opportunity for engineers, planners, managers and teams to try out ideas in advance of commitment to a course of action [10].

The goal of participative simulation, is to enable workers to exert direct influence over the product and process designs by bringing in their tacit knowledge, to combine it with expert knowledge, and to put the blend of both insights to the test. When these activities are supported by an attractive ICT interface, the resulting continuous improvement process may become intrinsically motivating for the work force[1]. Besides, it also contributes to the competitive advantage of the organization. Unfortunately, several problems hamper the introduction of participative simulation in manufacturing enterprises. The total number of users of simulation tools is still low. Another problem is how to generate a common description and let people with various experiences participate in the analysis. In addition, the tools or content-bases are not connected to the real world, and therefore, often reflect a state of the business, that is outdated.

In order to overcome these problems and accomplish that several tools and content bases are combined with the transparency of "a single multi-user tool", the PSIM project needs to develop several aspects: an extensible and modular ontology that will serve to facilitate interoperation and communication between applications and people; navigation functions in support of problem solving and decision scenarios, these functions must often be adapted to the changing needs of each company; and integration services for tools and systems including ERP (enterprise resource planning), and MES (manufacturing execution systems), ergonomic and sociotechnical applications. In this paper only the ontology is addressed in detail. The second Chapter describes the common process of ontology development. The third Chapter describes which additional aspects have been considered in the PSIM ontology development, and how.

\section{ONTOLOGY DEVELOPMENT}

An ontology corresponds in practice to a set of formal terms, usually with a hierarchical organisation, with associated formal definitions that specify their relationships with the other formal terms, and a set of constraints about their use in the knowledge representation of the domain studied. Each term 
can be seen as a knowledge category that can be instantiated[14]. General guidelines have been stated to help the ontology builders in their task. An ontology has to be rooted in a broad mutual understanding and agreement of the different domain stakeholders. The stakeholders have to agree on the general hypotheses made on the knowledge domain. These hypotheses deal with the different high level categories or concepts, which are used to express the objects or object types and their relations [12].

In a first step, an informal ontology is developed. Uschold [18] proposes to use two complementary processes to identify scope, terms and concepts of an informal ontology for an application domain: (1) identify motivating scenarios that can serve as the basis for defining a complete set of informal competency questions; the competency questions express different reasoning problems that must be supported; and (2) Use brainstorming to identify as many potentially important concepts as possible, and use trimming by grouping the concepts into various more or less distinct work areas such that terms within an area show more similarity in meaning, than between different areas (eg. terms related to activity, versus terms related to organisation); within each area, and for each term indicate the importance of including it in the ontology; remove the less important and/or the duplicated terms.

The resulting informal ontology serves as a specification for the subsequent formal code [18]. Setting a definition of terms used in an ontology and the choice of them is the most difficult task in the building of the ontology. Guidelines for choosing terms and defining them in a suitable way have been addressed by several authors $[11,14,17]$. Clarity is achieved by using examples to illustrate what is and what is not intended, and by stating all underlying assumptions, especially where they are not explicitly formalised in axioms. Consistency and coherence is achieved by avoiding circularity, by using terms that best conform to the common usage, by avoiding the introduction on unnecessary new terms. Dictionaries, thesauri, and technical glossaries should be consulted. Extensibility and reusability can be achieved by getting the right balance between being specific enough to perform the required tasks, but not so specific that it will be of little use to others; also by avoiding several terms that mean roughly the same thing. The authors recommend to work in a middle-out fashion rather than top-down or bottom-up: a bottom-up approach results in a very high level of detail and an increase of overall effort, it also makes it difficult to spot commonality between related concepts, and increases the risks of inconsistencies which leads in turn to re-work and yet more effort. To reach agreement when terms are used ambiguously, one should concentrate on the underlying ideas first, 
ignoring the terms, define each related idea, then decide on the most important ideas and lastly choose appropriate terms.

\section{ONTOLOGY FOR PARTICIPATIVE SIMULATION}

In addition to using the common ontology development techniques, an ontology for participative simulation in manufacturing enterprises, needs to leverage available bodies of generic or scientific knowledge "around" the domain. In the PSIM project, the following knowledge areas are addressed explicitly: reference architecture and methodology in enterprise modelling and integration; socio-technical systems design; ergonomy and the reduction of physical and mental workload; the improvement process and intrinsic motivation of employees; the development and deployment of ICT tools, especially ERP software packages, and their use of enterprise modelling techniques (IT vendors and IT consultants).

The PSIM ontology development gives each of these knowledge areas its due attention, as is explained below. An enterprise meta model based on CIMOSA[3] and European pre-standards [5,6,7] was taken as the source for the first core terms and their definitions. The completeness of this model was then checked by confronting it with the pertinent concepts related to the domains of socio-technical systems design, ergonomy, the PSIM procedure, and the AS-IS analysis at three pilot-sites. If a concept can not be explained or expressed with the help of the concepts in the model, it has to be added to the core. In a first step concepts have been formulated in natural language and translated to a semi formal one using the proposed model. By this way, we are building the first version of the ontology, the informal one.

\subsection{Reference Architecture and Methodology}

The relevance for participative simulation of reference architectures and methodology is derived from their relevance for integration projects as expressed in the report of the IFIP-IFAC task force[21]: “... a large part of integration projects [participative simulation projects] is in fact similar and common in every type of enterprise. Thus it could be captured, standardized and utilized instead of developing it again from scratch. Once standardized, generally accepted architectures can be supported by tools, methodologies, and a range of compatible products thus making the entire endeavour[ participative simulation] efficient in time and cost.... Methodologies usually are developed in conjunction with reference architectures..A methodology (for enterprise integration [or participative simulation]) is a set of proven guidelines, techniques, procedures which the 
end-user can follow in order to implement an enterprise integration policy as well as carry out integration [and change] projects within that policy."

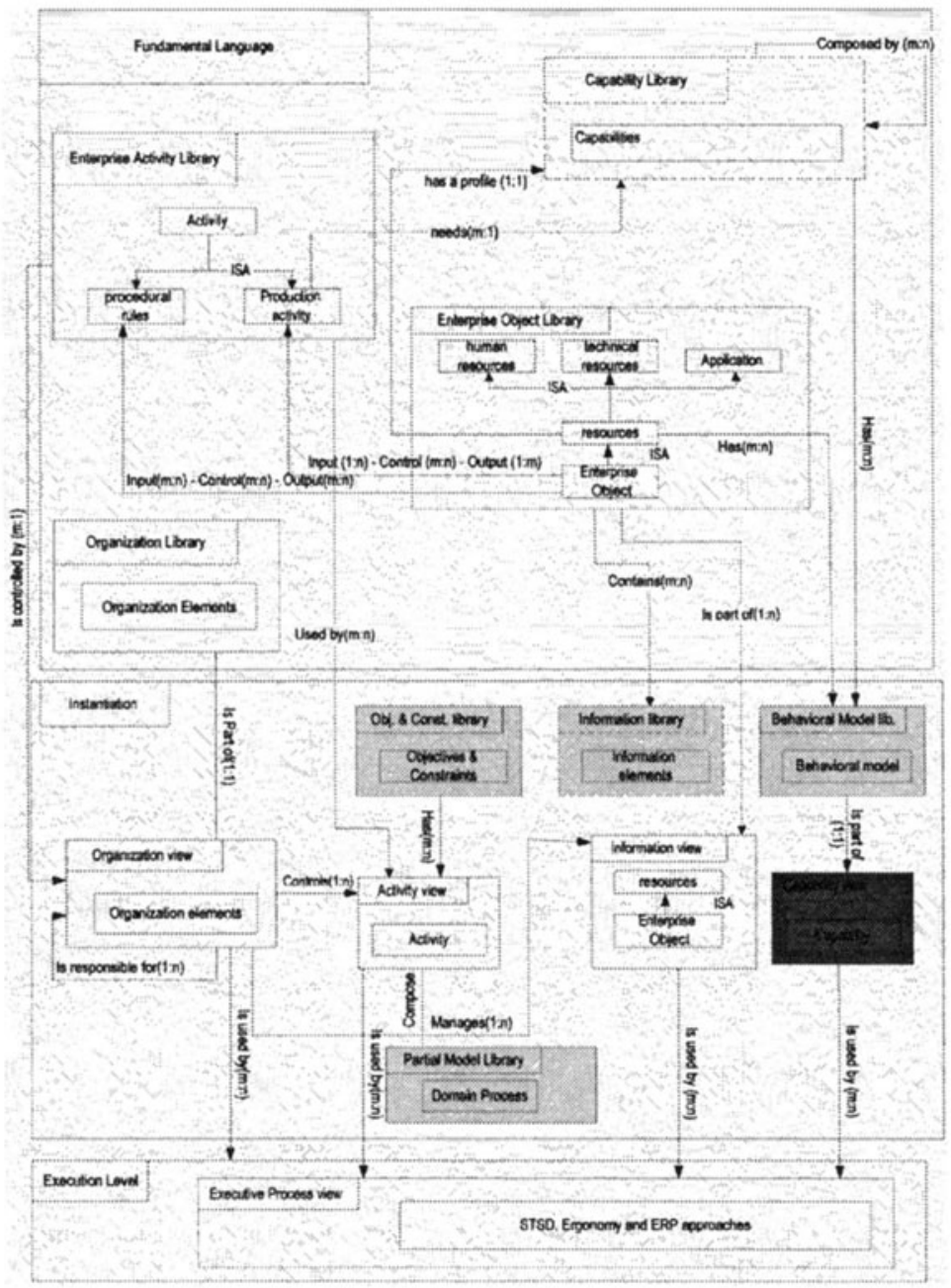

Figure 1. PSIM Draft Ontology Configuration Schema

The PSIM ontology draws on existing enterprise integration knowledge (CIMOSA [3], ENV 40003 [5], ARIS [17], DEM [19]), as well as on concepts for design and maintenance of enterprises for their entire life history (GERAM or Generalized Enterprise Reference Architecture and Methodology" [2,8]). The backbone of the participative simulation environment is an "ontology configuration schema" with a hierarchical structure that reflects the ENV 40003 step wise instantiation process. The ontology configuration schema is depicted in figure 1. Its aim is to support 
the initial configuration and further modification of the ontology for any particular enterprise.

The fundamental constructs at the "generic" level - the fundamental language (figure 1) - are three: activities, organization elements and enterprise objects. Their definition draws on the constructs defined in ENV 12204 [16]. Instances of these meta-constructs are created as template elements in libraries. Capabilities, at this level, serve as attributes, to support evaluations of whether or not production activities can be executed by or for the enterprise objects envisioned.

At the instantiation layer (figure 1), entities - organisation elements, activities and enterprise objects - are instantiated within three enterprise views: the organisation view, the activity view, and the information view covering the enterprise objects. When configuring the particular level model of the enterprise at the instantiation layer -- compare with the ENV 40003 particular level -- use is made of a number of "partial level" libraries: the partial model library, the objectives and constraints library, an information library and a behavioral model library. The capability view is not configured as such, since it results from choices made in the activity and information view. At this level, plenty of cross-view rules on enterprise configurations can be stated and enforced.

At the execution layer, the particular enterprise model configured at the instantiation layer, is blended with operational data, work orders and emergent problems to be solved. At this layer the enterprise model execution and integration services matter, as described in ENV 13354 EMEIS (Enterprise Modelling Execution and Integration Services [7]). Decision scenarios might cover the allocation of work to team members, the definition of the process plan for a new product, and so forth.

\subsection{Socio-technical Systems Design}

The social subsystem of worksystems has remained largely unrecognized in the modelling of manufacturing systems and the development of ICT applications. Reasons for this include: the overemphasis on computer technology to integrate enterprises; the lack of knowledge among ICT professionals of the scientific basis on which to model social and human factors; and the inadequacy for modelling social and human aspects of existing modelling techniques and methodologies.

Socio-Technical Systems Design (STSD) is concerned with the optimization and integration of the human factor in manufacturing systems, predominantly at the work group, departmental and organizational levels. It aims at improving the quality of work and organisation simultaneously through adaptation or fundamental redesign of the contents and composition 
of technology and human tasks [4]. Within the sociotechnical framework, a method was developed that specifically addresses the issue of allocating tasks between humans and technology, i.e. defining the degree of automation. Key to this method are design criteria on the level of work system, individual task, and human-machine interaction, which can be used also in system modelling and simulation [9].

\subsection{Ergonomy}

While the focus of the sociotechnical framework is the human-humantechnology interaction, the more specific aspects of fitting tasks and technology to human operators is dealt with by an ergonomic approach concerned with optimizing the tasks, technical systems and work stations in order to improve human performance and to reduce mental and physical workload. Data from the European Foundation for the Improvement of Living and Working Conditions indicate that a rise in 'time pressure' has taken place throughout Europe. Approximately $30 \%$ of the workers in the European Union are involved in painful and tiring postures for more than half of their working day and $40 \%$ of the workers are exposed to short repetitive tasks, which often lead to reduced quality, productivity, complaints or even sick leave. A recent survey reports on the workrelatedness of drop out from work due to psychological disfunctioning. Some important aspects in the reduction of workload are the good fit between task and personality, possibilities to develop and regulate your own work[20]. Therefore an important function in a participative simulation environment is envisioned that will warn users when unacceptable workload for humans and teams is anticipated in a particular work system design.

\subsection{Procedure}

Today improvements in companies are usually achieved, when problems occur and are successfully solved. The employees who deal with this task, are often motivated by the fear, that they will be called to account for the losses a problem causes to the company. Thus, the motivation for problemsolving activities is rather extrinsic with all its negative effects. Since it is not the attractiveness of the task but a coercion which induces the employees to solve problems, this activity lasts only as long as a pressure exists. In order to achieve continuous improvement the problem-solving has to motivate the employees intrinsically: the improvement process has to enrich the job contents and animate the work force to deal with it because of its attractiveness [15]. PSIM needs a generalized procedure for a situationindependent application. This procedure has to map the steps of an ideal 
improvement process considering an involvement of employees on all hierarchical levels.

The PSIM procedure contains the following steps: (1) Selection of the topic: Every employee can initiate an improvement process. That means that not only the worries of the management are dealt with, but even the concerns of the employees such as work overloading or occupational safety. Selection of participants: a problem classification scheme supports the identification of concerned company units. (3) Ontological convergence: During team sessions the current situation is described and it is stated why this situation is a problem. This step is supported by several tools, for instance process and product simulation. (4) Definition of goals: Based on the previous step the problem-solving team defines the goals. The goals are recorded by PSIM. (5) Idea generation: PSIM assists the idea generation by creativity conducive tools such as morphological matrix or Ishikawa diagrams. (6) Simulation of the ideas: relevant simulation tools are selected from those available in the environment, to simulate the effects of the different ideas. (7) Evaluation: results of the simulations, both improvements and possible negative effects, are evaluated and presented. (8) Selection of solution: based on the results of the previous step and relevant financial criteria the team selects a solution in cooperation with supervisors. (9) The implementation of the chosen solution is supported by PSIM.

The above description of the procedure implies a further important component of PSIM: the moderator function. The simulation platform has to support the problem-solving process by informing the participants about meetings, coordinating possible dates, sending reminders about workpackages which have to be done et cetera. The moderation is necessary to keep the process going on, even when the work load endangers the progress.

\subsection{Tools}

A final challenge is the integration of various simulation and other tools in the participative simulation environment. On the one hand these tools have to be connected to the different steps of the procedure, as it is applied in variable work contexts. On the other hand the tools have to be linked to the users with a user-friendly interface. Also it must be possible for the tools and systems to exchange data, results of their applications, as well as data on the state of the enterprise. These tasks should not be underestimated, because of existing tools and systems have considered software ergonomic aspects and data exchange to a very various extent, because of the short cycle time of some software tools, and the frequent occurrence of in-house developed IT systems. 
PSIM will provide a structure which facilitates an easy plug-in of other than the originally integrated tools. For certain tools, this plug-in has to be relative to the particular ontology that has been configured for the enterprise deploying the tool.

\section{CONCLUSION AND FUTURE WORK}

To develop an ontology for participative simulation the common ontology development techniques have been combined in order to leverage available knowledge in areas such as enterprise architectures, ergonomy, socio-technical systems, change procedures, and IT tool development and integration. The PSIM approach and domain choices have been presented in this paper. The draft ontology configuration schema that has been presented will be subjected to depth, scope and particularization tests during the coming months, so that it evolves towards the kernel of a participative simulation environment that can easily be expanded and deployed in multiple organisations, for multiple user profiles and use-scenarios.

Further developments also include capture of the ontology in UML/XML schemas, and the building of interfaces for navigator, tools and users. Towards the end of the PSIM project, the participative simulation environment will be tested at three pilots.

\section{ACKNOWLEDGEMENT}

This research has been carried out in the EU IST-IMS PSIM Project

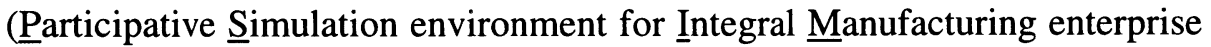
renewal (IMS 1999-00004). PSIM is part of HUMACS, a project within the international IMS research program.

\section{REFERENCES}

[1] Berg, R.J. van den, Eijnatten, F.M. van, Vink, P., \& Goossenaerts, J.B.M., Leveraging human capital in assembly organizations: The case for participative simulation. Proceedings IST conference. Helsinki, 1999.

[2] Bernus, P. and L. Nemes, editors. Modelling and Methodologies for Enterprise Integration. Chapman \& Hall, London, UK, 1996.

[3] CIMOSA, ESPRIT Consortium AMICE, editor. CIMOSA: Open System Architecture for CIM. Springer Verlag, Berlin, 2nd, rev. and ext. ed edition, 1993.

[4] Eijnatten, F.M. van, \& Zwaan, A.H. van der. The Dutch IOR approach to organizational design: An Alternative to Business Process Re-engineering? Human Relations, 1998, 51 (3), 1-30. 
[5] ENV 40 003. CEN/CENELEC/IT/WG ARC. Computer integrated manufacturing -systems architecture -- framework for enterprise modelling. European prestandard, CEN/CENELEC, 1990.

[6] ENV 12 204. CEN TC310 WG1. Computer integrated manufacturing -- systems architecture -- constructs for enterprise modelling. European prestandard, CEN/CENELEC, 1996.

[7] ENV 13354. CEN TC310. Computer integrated manufacturing -- systems architecture Enterprise Modelling Execution and Integration Services, European prestandard, CEN/CENELEC, 1999.

[8] IFIP-IFAC Task Force. GERAM: Generalised Enterprise Reference Architecture and Methodology. Version 1.6.1, May 1998.

[9] Grote, G. A participatory approach to the complementary design of highly automated work systems. In: G. Bradley \& H.W. Hendrick (Eds.), Human factors in organizational design and management - IV (pp.115-120). Amsterdam: Elsevier, 1994.

[10] Groumpos P, Krauth J. Simulation in Industrial Manufacturing: Issues and Challenges, In: Fichtner D, Mackay R. Facilitating deployment of Information and Communications Technologies for competitive manufacturing. Dresden: The European Conference on Integration in Manufacturing - IiM ,1997.

[11] Gruber, T. Toward principles for the design of ontologies used for knowledge sharing. (ed. N. Guarino; Proc. of the International Workshop on Formal Ontology, Padova, Italy, 1992.

[12] Guarino N., M. Carrara M., P. Giaretta, Formalizing Ontological Commitments. In Proc. Of AAAI'94, Seattle, Washington, 31 july-4 august 1994.

[13] Heller, F., Pusic, E., Strauss, G., \& Wilpert, B., 'Organizational participation: Myth and reality'. Oxford University Press, 1998.

[14] Martin P. (1996) Exploitation de graphes conceptuels et de documents structurés et hypertextes pour l'acquisition de connaissances et la recherche d'informations, Ph.D., Nice-Sophia Antipolis , France.

[15] Orban, P. \& Völker, S., How to Improve Working Processes through Collaboration in Cross-Departmental Teams. In: P. Vink, E.A.P. Koningsveld \& S. Dhondt (Eds.), Human Factors in Organizational Design and Management - VI, Den Haag: Elsevier Science 1998.

[16] Reyneri, C., Operational Building Blocks for Process Modelling, Enterprise Engineering and Integration Standardisation Workshop, Berlin, May 24-26, 2000.

[17] Scheer, A.-W.. Business Process Engineering -- Reference Models for Industrial Enterprises. Springer Verlag, Berlin, 1994.

[18] Uschold M., M. King, S. Moralee, and Y. Zorgios (1998) The enterprise ontology. The Knowledge Engineering Review, 13.

[19] Van Es, R.M. and Post, H.A. 1996, Dynamic Enterprise Modelling: linking business and $I T$, Kluwer, Deventer, the Netherlands.

[20] Vink P, Peeters, M. Balancing organizational, technological and human factors: the vision of production management. In: Vink P, Koningsveld EAP, Dhondt S, eds. Human factors in Organizational Design and Management VI. London: Elsevier Science Ltd, London, 1998:7-11.

[21] Williams, T.J., P. Bernus, J. Brosvic, D. Chen, G. Doumeingts, L. Nemes, J.L. Nevins, B. Vallespir, J. Vlietstra, and D. Zoetekouw. Architectures for integrating manufacturing activities and enterprises. Computers in Industry, 24/2-3, 1994. 